\title{
Precise Reflection Coefficient Measurements With an Untuned Reflectometer
}

\author{
W. E. Little* and D. A. Ellerbruch* \\ Institute for Basic Standards, National Bureau of Standards, Boulder, Colo.
}

(March 9, 1966)

\begin{abstract}
The precision tuned reflectometer technique of reflection coefficient measurement is, at present, the most accurate technique available. Utilization of the technique requires the use of tuners and sliding terminations to eliminate or reduce the inherent errors of a directional coupler.

This paper describes a reflection coefficient measurement technique that approaches the accuracy of the tuned reflectometer. Instead of tuners, the technique uses an ideal one-quarter wavelength waveguide section to eliminate the measurement error introduced by finite directional coupler directivity and to reduce to second order the error introduced by an equivalent generator mismatch.

A comparison of the quarter-wave technique with the tuned reflectometer technique through the measurement of the reflection coefficient of three $9 / 16$ inch coaxial waveguide terminations is also included.
\end{abstract}

Key Words: Microwave, impedance, measurements, reflectometer.

\section{Introduction}

The reflectometer technique for microwave reflection coefficient measurements is now widely used [1]. ${ }^{1}$ The measurement accuracy of a basic reflectometer (a simple directional coupler coupled to the reflected wave) depends on the directivity of the directional coupler and the equivalent generator mismatch. The best known method of improving the measurement accuracy of the basic reflectometer requires the use of tuners, tuning procedures, and auxiliary equipment $[2,3]$. The result of this tuning is an increase in the effective directivity of the coupler and a matched equivalent generator. At present, the most accurate measurements are achieved with this tuned reflectometer.

The purpose of this paper is to present another method of improving the measurement accuracy of the basic reflectometer technique. This method utilizes a quarter-wavelength . waveguide section. Proper use of the quarter-wave section during the measurement procedure will, in theory, eliminate the error caused by a finite directivity and will reduce the error caused by a mismatched equivalent generator to second order.

No detailed error analysis is included in this paper; however, a laboratory measurement comparison with the tuned reflectometer indicates that the total measurement error is small. Such an error analysis may be given at a later date, depending on results of further laboratory experiments with the method.

* Radio Standards Engineering Division. National Bureau of Standards, Boulder, Colo.

${ }^{1}$ Figures in brackets indicate the literature references at the end of this paper.

\section{Theory}

The operation of a reflectometer is discussed in detail elsewhere [3]. Therefore, only a brief review will be presented here as a starting point for the discussion of the quarter wavelength technique. The side-arm output, $b_{3}$, of a directional coupler connected to sample the reflected wave (see fig. 1) can be expressed as

$$
b_{3}=k b_{G} \frac{\frac{1}{K}+\Gamma_{T}}{1-\Gamma_{2 i} \Gamma_{T}},
$$

where $b_{G}$ is the component of the incident wave furnished by the generator, $\Gamma_{2 i}$ and $k$ are both functions of the scattering coefficients of the directional coupler and reflection coefficients of the generator and detector, and $\Gamma_{T}$ is the reflection coefficient of the terminating element. The symbol $\Gamma_{2 i}$ represents the equivalent source reflection coefficient presented to the reflector under study at the reference plane. The symbol $K$ is approximately equal to the directivity ratio of the directional coupler.

The desired performance of the directional coupler is realized when $\Gamma_{2 i}=0$ and $1 / K=0$. Equation (1) then reduces to

$$
b_{3}=k b_{G} \Gamma_{T} .
$$

Hence, inspection of (1) shows that the terms involving $1 / K$ and $\Gamma_{2 i}$ introduce first order errors in $\Gamma_{T}$, when $\Gamma_{T}$ is determined according to (2) from a measurement of 
the side-arm output, $b_{3}$, of an untuned $\left(1 / K\right.$ and $\left.\Gamma_{2 i} \neq 0\right)$ directional coupler. Reference 3 discusses the minimization of these errors by proper use of tuners.

It is possible to eliminate the term $1 / K$ and simultaneously reduce the error arising from a finite $\Gamma_{2 i}$ to second order by a measurement procedure utilizing an ideal waveguide section that is one-quarter wavelength long. The theoretical basis for the method is as follows:

If the unknown is connected directly to the reference plane of the untuned reflectometer shown in figure 1 , the side-arm output, $b_{1 \mu}$, is

$$
b_{1 \mu}=k b_{c} ; \frac{\frac{1}{K}+\Gamma_{\mu}}{1-\Gamma_{2 i} \Gamma_{\mu}}
$$

where $\Gamma_{\mu}$ is the reflection coefficient of the unknown.

Interposing a precision quarter-wavelength waveguide section between the reflectometer and $\Gamma_{\mu}$ adds a $180^{\circ}$ phase shift to the argument of the impedance appended to the reference plane, causing the reflection coefficient at this plane to be $-\Gamma_{\mu}$. With the quarter-wavelength section inserted between the reference plane and the unknown, as shown in figure 2 , the side-arm output, $b_{2 \mu}$, is

$$
b_{2 \mu}=k b_{c_{i}} \frac{\frac{1}{K}-\Gamma_{\mu}}{1+\Gamma_{2 i} \Gamma_{\mu}} .
$$

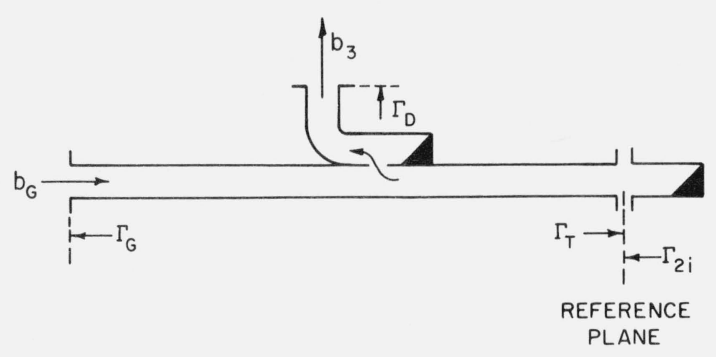

FigURE 1. A basic reflectometer.

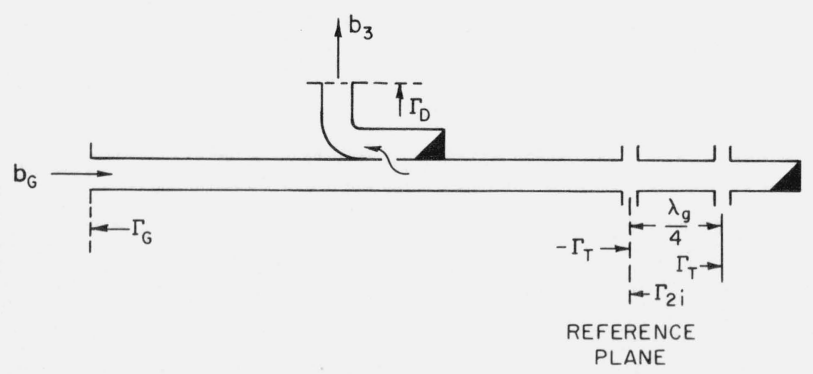

FiguRE 2. A basic reflectometer with a quarter-wave section connected.
The difference between $b_{1 \mu}$ and $b_{2 \mu}$ is then

$$
b_{1 \mu}-b_{2 \mu}=2 k b_{G} \Gamma_{\mu} \frac{1+\frac{1}{K} \Gamma_{2 i}}{1-\left(\Gamma_{2 i} \Gamma_{\mu}\right)^{2}} .
$$

If the unknown is now replaced with a quarterwavelength standard short circuit ${ }^{2}$ whose reflection coefficient is unity and the above measurement procedure repeated, formula (5), upon substitution of $\Gamma_{s}=1$ for $\Gamma_{\mu}$, reduces to

$$
b_{1 s}-b_{2 s}=2 k b_{G} \frac{1+\frac{1}{K} \Gamma_{2 i}}{1-\left(\Gamma_{2 i}\right)^{2}},
$$

where $b_{1 s}$ is the sidearm output when the standard short circuit is connected directly to the reference plane, and $b_{2 s}$ is the sidearm output when the ideal quarter-wavelength section is placed between the reference plane and the short circuit.

Dividing (6) by (5) results in

$$
\frac{b_{1 s}-b_{2 s}}{b_{1 \mu}-b_{2 \mu}}=\frac{1}{\Gamma_{\mu}} \frac{1-\left(\Gamma_{2 i} \Gamma_{\mu}\right)^{2}}{1-\Gamma_{2 i}{ }^{2}} .
$$

Note that (7) does not contain the $1 / K$ factor and that $\Gamma_{2 i}$ occurs only to the second power. $\left|\Gamma_{2 i}\right|$ is usually less than 0.05 in a practical situation; hence, $\left|\Gamma_{2 i}\right|^{2}$ $\ll 1$. Therefore, the limitations of the untuned directional coupler (i.e., finite directivity and a mismatched equivalent generator) have been essentially circumvented, and the correct value of $\Gamma_{\mu}$ can be determined without tuning the coupler.

To evaluate $\Gamma_{\mu}$ by means of (7), it appears that four individual (complex) quantities have to be measured. If one is interested only in a magnitude measurement, which is usually the case, a relatively simple measurement procedure can be used to determine the ratio $\left|b_{1 s}-b_{2 s}\right| /\left|b_{1 \mu}-b_{2 \mu}\right|$ directly, in decibels.

\section{Measurement Procedures for Determining the Modulus of $\Gamma_{\mu}$}

A measurement system has been constructed and used to substantiate the quarter-wave method experimentally. A block diagram of the system is shown in figure 3. The measurement procedure is as follows:

1. One end of the precision quarter-wavelength section of waveguide is connected to the reference plane and terminated with a standard short circuit. The signal from the side arm under these conditions is $b_{2 s}$ of formula (7).

\footnotetext{
${ }^{2} \mathrm{~A}$ quarter-wavelength standard short circuit is a quarter wavelength of precision line terminated by a shorting metal wall. If a simple shorting plate is used, $\Gamma_{s}=-1$ is substituted for $\Gamma_{\mu}$ in (5) and one side of $(7)$ would be multiplied by a -1 .
} 


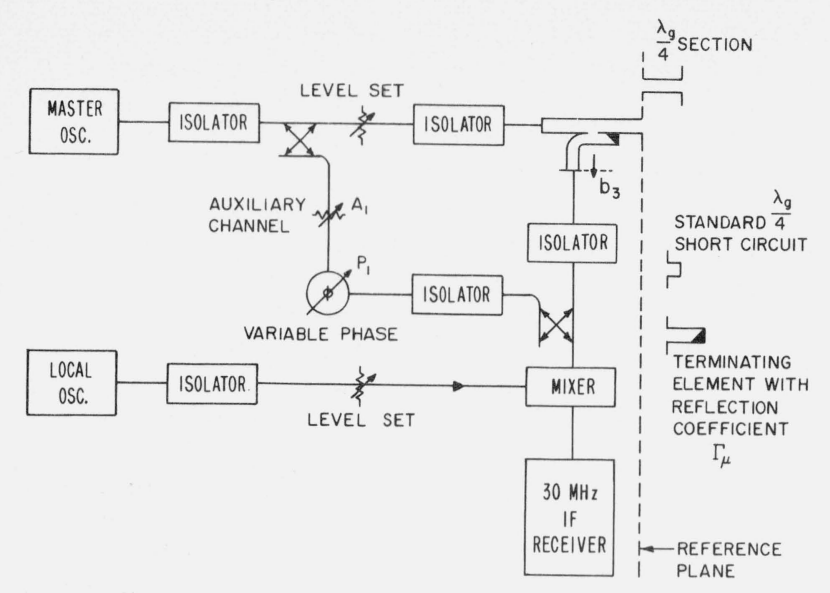

FigURE 3. Block diagram of quarter-wavelength measurement system.

2. The variable phase shifter and attenuator, designated $P_{1}$ and $A_{1}$ in figure 3 , are adjusted to produce a null at the $30 \mathrm{MHz}$ IF receiver. This adjustment sets the signal from the auxiliary channel, $b_{1 A}$, equal to $-b_{2 s}$.

3. The ideal quarter-wavelength section is removed and the standard short circuit is connected directly to the reference plane. Under these conditions the signal from the side arm is $b_{1 s}$ of (7). Because the signal from the auxiliary channel of figure 3 was adjusted in the preceding step to produce the signal $-b_{2 s}$, the receiver, which responds to the sum of the outputs of the side-arm and auxiliary channel, registers $\left|b_{1 s}-b_{2 s}\right|$.

4. The standard $30 \mathrm{MHz}$ attenuator in the IF receiver is adjusted to produce an arbitrary reference level at the indicator.

5. The short circuit is removed from the reference plane, and the quarter-wavelength section is connected and terminated in the unknown reflection coefficient. Under these conditions the signal out of the sidearm is equal to $b_{2 \mu}$ of (7).

6. The phase shifter and attenuator $\left(P_{1}\right.$ and $\left.A_{1}\right)$ are now readjusted to again produce a null at the $30 \mathrm{MHz}$ receiver. This adjustment provides that $b_{A 2}=-b_{2 \mu}$, where $b_{A 2}$ is the signal from the auxiliary channel.

7. The quarter-wavelength section is removed, and the unknown is connected directly to the reference plane. The signal into the $30 \mathrm{MHz}$ IF receiver is again the sum of the signals from the side-arm and auxiliary channel, i.e., $\left|b_{1 \mu}-b_{2 \mu}\right|$. A record is made of the change (in decibels) in the setting of the standard $30 \mathrm{MHz}$ attenuator in the receiver required to return the indicator to the reference level chosen in step four. This difference (in decibels) between the final attenuator setting and the setting at step four is a measure of the ratio $\left|b_{1 s}-b_{2 s}\right| /\left|b_{1 \mu}-b_{2 \mu}\right|$. Thus,
$\mathrm{dB}($ measured $)=20 \log \left|\frac{b_{1 s}-b_{2 s}}{b_{1 \mu}-b_{2 \mu}}\right|$

$$
=20 \log \frac{1}{\left|\Gamma_{\mu}\right|} \frac{\left|1-\left(\Gamma_{2 i} \Gamma_{\mu}\right)^{2}\right|}{\left|1-\Gamma_{2 i}^{2}\right|} .
$$

Assuming that $\left|\Gamma_{2 i}^{2}\right| \leqslant 1$, this equation reduces to approximately

$$
\mathrm{dB}(\text { measured })=20 \log \frac{1}{\left|\Gamma_{\mu}\right|},
$$

whence the unknown reflection coefflcient magnitude is given by

$$
\left|\Gamma_{\mu}\right|=10-\frac{\mathrm{dB}(\text { measured })}{20} .
$$

\section{Measurement Results}

The reflection coefficient magnitude of three 9/16 inch coaxial terminations were measured at $4 \mathrm{GHz}$ using the quarter-wave technique. These measurements were compared with those obtained with a tuned reflectometer and those obtained with an untuned reflectometer that did not utilize a quarterwavelength section. Table 1 summarizes the results.

The first column lists the values obtained for the three terminations when measured with a tuned reflectometer. The tolerances associated with these values include the measurement error as well as the non-repeatability obtained by rotating the termination. In the second column are listed the VSWR's corresponding to the reflection coefficients in the first

\begin{tabular}{|c|c|c|c|c|c|}
\hline \multicolumn{2}{|c|}{$\begin{array}{l}\text { TUNED REFLECTOMETER } \\
\text { MEASUREMENT VALUES }\end{array}$} & \multicolumn{2}{|c|}{$\begin{array}{l}\text { VALUES OBTA INED WITH } \\
\text { IMPERFECT COUPLER }\end{array}$} & \multicolumn{2}{|c|}{$\begin{array}{l}\text { VALUES OBTAINED WITH } \\
\text { IMPERFECT COUPLER } \\
\text { AND } 1 / 4 \lambda \text { SECTION }\end{array}$} \\
\hline$|\Gamma|$ & VSWR & $|\Gamma|$ & VSWR & $|\Gamma|$ & VSWR \\
\hline $\begin{array}{r}0.0217 \\
\pm 0.0006\end{array}$ & $\begin{array}{r}1.0443 \\
\pm 0.0012\end{array}$ & 0.042 & 1.088 & 0.0224 & 1.0458 \\
\hline $\begin{array}{r}0.0902 \\
\pm 0.0015\end{array}$ & $\begin{aligned} & 1.1983 \\
\pm & 0.003\end{aligned}$ & 0.108 & 1.242 & 0.0918 & 1.2022 \\
\hline $\begin{array}{r}0.3334 \\
\pm 0.0028\end{array}$ & $\begin{array}{r}2.000 \\
\pm 0.006\end{array}$ & 0.351 & 2.082 & 0.3353 & 2.0089 \\
\hline
\end{tabular}
column.

TABLE 1. Tabulated measurement results

In the third and fourth columns are listed the reflection coefficients and VSWR's obtained with an untuned directional coupler but no quarter-wavelength section. These values were obtained as follows: The standard short circuit was connected to the reference plane, and an indicator reference point 
was obtained. The short circuit was then replaced by the unknown impedance, and the reflection coefficient was computed from the measured change in the signal level at the indicator. These values are subject to all the errors due to imperfections in the directional coupler. The directional coupler employed in the three systems with which the data of table 1 were obtained (including that of fig. 3), had a directivity of approximately $30 \mathrm{~dB}$ (this corresponds to a $1 /|K|$ value of approximately 0.032 ) and a $\left|\Gamma_{2 i}\right|$ of less than 0.02 .

In the fifth and sixth columns of table 1 are listed the reflection coefficient magnitude and VSWR values obtained by using the quarter-wavelength measurement technique described in this report.

The values obtained using the quarter-wave technique are in near agreement with the values obtained using the tuned reflectometer. The values obtained with the untuned reflectometer when the quarterwave section was not used are considerably in error.

\section{Conclusions}

Precise reflection coefficient measurements are possible with an untuned reflectometer if the quarterwave measurement technique is used. The theory was verified experimentally by measuring the reflector coefficient magnitudes of several terminations with a tuned reflectometer and then remeasuring them using the quarter-wave technique with an untuned reflectometer. These same terminations were also measured directly with an untuned reflectometer without application of the quarter-wavelength technique, to demonstrate the improvement in accuracy provided by the quarter-wave method.

Relative phase data were neglected during this evaluation. No serious difficulties are anticipated in modifying the measurement system to obtain phase data so that the quarter-wave technique will then provide the complex reflection coefficient.

Use of the quarter-wave technique instead of a tuning procedure eliminates the need for variable-phase high- and low-reflection loads and shortens the required length of precision line. The most important potential application of the quarter-wave technique is in the measurement of impedance at frequencies below approximately $1 \mathrm{GHz}$. With decreasing frequency the tuned reflectometer becomes increasingly cumbersome.

\section{References}

[1] Ginzton, E. L., Microwave Measurements, p. 300 (McGrawHill Book Co., Inc., New York, N.Y., 1957).

[2] Engen, G. F., and R. W. Beatty, Microwave reflectometer techniques, IRE Trans. MTT MTT-7, No. 3, 351-355 (July 1959).

[3] Anson, Wilbur J., A Guide to the use of the modified reflecmeter technique of VSWR measurement, J. Res. NBS 65C (Engr. and Instr.) No. 4, 217 (Oct.-Dec. 1961).

(Paper 70C3-227) 